\title{
International Investment (Trade) Factor and Its Effect on GDP: BRICS Case Study
}

\author{
Prof. Dr. Ercan Ekmekçioğlu \\ Dr. İsmail Çelik \\ Mevlana (Rumi) University \\ eekmekcioglu@mevlana.edu.tr \\ ismailce98@gmail.com
}

Abstract

Purpose: The purpose of the study is to assess the degree to which International Investment (Trade) Factor has impacted on GDP looking at BRICS region. The title is dubbed: "International Investment (Trade) Factor and its Effect on GDP: BRICS Case Study". Design/Methodology/Approach: The research adopted a desk research approach. in this regard, resource materials were developed from economic journals, books, articles, policy documents, and other credible websites just to a mention a few. Findings and assumption: The finalised research assessed the contribution trade has had to GDP in BRICS region. Trade has been considered as a fundamental growth factor in BRIC and an accelerating component which has been of great attention within the region. The current research argues that trade has benefited BRICS economy in terms of technology, skilled labour, consumer spending, total investment by companies, total government spending and balance of trade. The analysis bases on data collection from the period 2010-2013 so as to test the relationship between the two economic indicators. As indicated, the main research variables included FDI inflows, population growth, exports, and GDP. for data analysis, the researcher examines the descriptive statistics, correlation, and regression model using Microsoft Excel 2013. The main dependent variable being "trade"; it was anticipated that the trade function in regression model and other statistical tests would have positive relationship with other GDP variables. Research limitations/Implications: The main future research undertaking would be to use quantitative measures so as to establish the correlation between Trade as an international investment process and GDP dynamics.Practical Implications: The engagement in economic growth through increased GDP would be determined by the extent of international trade dynamics in BRICS region. Originality/Value: This research paper addresses fundamental policy issues that may be considered in BRICS region on how best to enhance international investment (trade) so as to support the rest of the economy.

Keywords: BRICS, Trade, International Investment, GDP, Trade, Exports.

\section{Introduction}

The purpose of the study is to assess the degree to which International Investment (Trade) Factor has impacted on GDP in BRICS region. Better still; the analysis looks at the role of exports on GDP growth within BRICS region. It is so that there are many other aspects supporting GDP growth in this region and trade is just one of the sectors. However, such investigation is still significant in the sense it will inform the reader whether trade has had any valuable place in the ongoing economic growth within BRICS region. BRICS region incorporates Brazil, Russia, India, China, and South Africa. This is a region that has been undergoing tremendous economic growth since its inception (Smith, 2011). The gross domestic product (GDP) serves as the most basic measure of a country's economic health (Liu et al, 2002). Further, GDP constitutes the monetary value of the entire finished goods or services produced by a given country in a given period of time (Liu et al, 2002). GDP is the total value for consumer spending, government outlays (spending), investments and balance of trade for a country (Makki \& Somwap, 2004). Therefore, from the understanding of GDP and its variables one can see that trade (balance of trade) takes just a part of it meaning it is not enough to explain GDP growth. However, just to remind the reader in this work the aim is to show whether BRICS region has benefited from export trade in enhancing its GDP growth. What has been the relationship? in the past, scholars developed models aimed at demonstrating the relationship involved between exports and economic growth (Sharma \& Panagiotidis, 2005). for instance, the export-led growth (ELG) model is one of them and secondly, growth-driven export model. in the former case, it affirms that comparative advantage catalyses trade activity consequently resulting in productive as well as efficient utilization of resources (Thangavelu \& Rajaguru, 2004). Thus when the export sector increases other improvements follow such as improved technology, availability of capital, and other growth (Rashid, 2007). Other models that have been used refers to import-led growth (ILG) or growth driven export-import (GDE/GDI) (Ram, 2004). in this current work the researcher shall basically focus on assess the potential causal relationship between GDP, imports, and exports to be estimated at current prices from the period 20042013. The main tests will include: 
i. The correlation between GDP and exports among the BRICS partners

ii.Export cause growth to GDP among the BRICS partners

\section{Methods}

In this analysis, the aim shall be to critically evaluate the relationship between GDP growth and exports for the BRICS region. However, due to the limitations embedded in the type of data that shall be used, the researcher will employ a simpler model to demonstrate the basic relationship among the variables underscored earlier. The general scope of production (dependent variable) will be featured in the production function as outlined in the equation below:

$Y=f(L, K, X)$

Thus;

$L=$ Labour input

$\mathrm{K}=$ Capital Input

$X=$ Level of exports

$Y=$ Aggregate real output

A growth equation may be developed in light of the equation, as provided by Rati Ram, for example:

$Y^{\prime}=\beta L L^{\prime}+a K(I / Y)+\beta X X^{\prime}$

$L=$ Rate of labour's growth

$X^{\prime}=$ Rate of export's growth

$Y^{\prime}=$ Growth rate

$I / Y=$ Investment-output ratio

However, over the same equation the researcher may introduce a constant term as well as a stochastic component to the same; this will be as follows:

$Y=\beta 0+\beta L L^{\prime}+\alpha K(I / Y)+\beta X X^{\prime}+u$

In this work the model to be used is more inclined to exogenous growth model since the researcher shall not use initial GDP variable, government spending, consumer spending, or research and development. The mentioned variables are fundamental to the endogenous growth model though in this work it was better to rely on exogenous growth model. The researcher is persuaded by Foster's equation to develop a regression model shown below:

$\Delta \operatorname{lnGDPt}=\beta 1 \mathrm{INVt}+\beta 2 \Delta \mathrm{POPt}+\beta 3 \Delta \operatorname{lnEXPt}+\mathrm{c}$

$c=$ constant

INV = Investment (FDI inflows)

$\mathrm{POP}=$ Population

Exp $=$ Exports

It is worth mentioning the study did not reply on panel data but rather analysed each BRICS country separately. The researcher hypothesizes a negative coefficient between growth and investment variable; the other hypothesis is that the relationship between growth and exports and population shall constitute a positive coefficient. The main data shall be obtained from the World Bank so as to have consistency and reliability/validity of data. The software tool carried out to perform statistical models was excel 2013.

\section{Results}

To start with is profiling the GDP per capita for BRICS region individually as nations. The data has been obtained from World Bank data base for each country in the period 2004-2012. However, it will be consolidated as shown in table 1 in the appendix. Now the next thing is to illustrate the manner in which other economic indicators in every region have supported GDP per capita.

Exhibit I: Regression output for South Africa 
SUMMARY OUTPUT

\begin{tabular}{|c|c|}
\hline \multicolumn{2}{|c|}{ Regression Statistics } \\
\hline Multiple R & $\begin{array}{l}0.95776 \\
0983\end{array}$ \\
\hline R Square & $\begin{array}{l}0.91730 \\
61\end{array}$ \\
\hline $\begin{array}{l}\text { Adjusted R } \\
\text { Square }\end{array}$ & $\begin{array}{l}0.85528 \\
5675\end{array}$ \\
\hline $\begin{array}{l}\text { Standard } \\
\text { Error }\end{array}$ & $\begin{array}{l}387.754 \\
3494\end{array}$ \\
\hline $\begin{array}{l}\text { Observatio } \\
\text { ns }\end{array}$ & 8 \\
\hline
\end{tabular}

ANOVA

\begin{tabular}{llllll}
\hline & df & SS & MS & F & $\begin{array}{l}\text { Significa } \\
\text { nce F }\end{array}$ \\
\hline Regression & 3 & 6671356. & 2223785 & 14.790 & 0.01246 \\
& & 601 & .534 & 3872 & 268 \\
Residual & 4 & 601413.7 & 150353. & & \\
& & 418 & 4354 & & \\
Total & 7 & 7272770. & & & \\
\hline
\end{tabular}

\begin{tabular}{|c|c|c|c|c|c|c|c|c|c|}
\hline & & $\begin{array}{l}\text { Coefficie } \\
\text { nts }\end{array}$ & $\begin{array}{l}\text { Standard } \\
\text { Error }\end{array}$ & t Stat & $\begin{array}{l}\mathrm{P}- \\
\text { value }\end{array}$ & $\begin{array}{l}\text { Lower } \\
95 \%\end{array}$ & $\begin{array}{l}\text { Upper } \\
95 \%\end{array}$ & $\begin{array}{l}\text { Lower } \\
95.0 \%\end{array}$ & $\begin{array}{l}\text { Upper } \\
95.0 \%\end{array}$ \\
\hline & Intercept & $\begin{array}{l}- \\
63594.5 \\
9432\end{array}$ & $\begin{array}{l}34465.15 \\
766\end{array}$ & $\begin{array}{l}- \\
1.84518 \\
5069\end{array}$ & $\begin{array}{l}0.1387 \\
6168\end{array}$ & $\begin{array}{l}- \\
159285 . \\
2126\end{array}$ & $\begin{array}{l}32096 . \\
024\end{array}$ & $\begin{array}{l}- \\
159285 . \\
2126\end{array}$ & $\begin{array}{l}32096.0 \\
24\end{array}$ \\
\hline $\begin{array}{l}\text { Invest } \\
\text { ment }\end{array}$ & $\begin{array}{l}701422007 . \\
6\end{array}$ & $\begin{array}{l}- \\
9.20654 \\
\text { E-08 }\end{array}$ & $\begin{array}{l}5.21796 \\
\text { E- } 08\end{array}$ & $\begin{array}{l}- \\
1.76439 \\
5742\end{array}$ & $\begin{array}{l}0.1524 \\
3288\end{array}$ & $\begin{array}{l}- \\
2.36939 \\
E-07\end{array}$ & $\begin{array}{l}5.2808 \\
\text { E-08 }\end{array}$ & $\begin{array}{l}- \\
2.36939 \\
\mathrm{E}-07\end{array}$ & $\begin{array}{l}5.2808 \mathrm{E} \\
-08\end{array}$ \\
\hline $\begin{array}{l}\text { Export } \\
s\end{array}$ & $\begin{array}{l}578897524 \\
92\end{array}$ & $\begin{array}{l}2.5952 E \\
-08\end{array}$ & $\begin{array}{l}1.60546 \\
E-08\end{array}$ & $\begin{array}{l}1.61648 \\
1362\end{array}$ & $\begin{array}{l}0.1812 \\
9623\end{array}$ & $\begin{array}{l}- \\
1.86228 \\
E-08\end{array}$ & $\begin{array}{l}7.0527 \\
\text { E-08 }\end{array}$ & $\begin{array}{l}- \\
1.86228 \\
E-08\end{array}$ & $\begin{array}{l}7.0527 \mathrm{E} \\
-08\end{array}$ \\
\hline $\begin{array}{l}\text { Popula } \\
\text { tion }\end{array}$ & $\begin{array}{l}1.30627464 \\
5\end{array}$ & $\begin{array}{l}51298.4 \\
5391\end{array}$ & $\begin{array}{l}26952.60 \\
682\end{array}$ & $\begin{array}{l}1.90328 \\
3577\end{array}$ & $\begin{array}{l}0.1297 \\
4658\end{array}$ & $\begin{array}{l}- \\
23533.9 \\
7936\end{array}$ & $\begin{array}{l}126130 \\
.887\end{array}$ & $\begin{array}{l}23533.9 \\
7936\end{array}$ & $\begin{array}{l}126130 . \\
887\end{array}$ \\
\hline
\end{tabular}

Source: (Calculation by Author)

Exhibit II: Regression output for Russian Federation 
SUMMARY OUTPUT

\begin{tabular}{ll}
\hline Regression Statistics \\
\hline Multiple R & 33 \\
& 0.996099 \\
R Square & 0.992213 \\
Adjusted & 0.986374 \\
R Square & 282 \\
Standard & 353.4515 \\
Error & 192 \\
Observati & \\
ons & 8 \\
\hline
\end{tabular}

ANOVA

\begin{tabular}{llllll}
\hline & df & SS & MS & F & $\begin{array}{l}\text { Significa } \\
\text { nce } \mathrm{F}\end{array}$ \\
\hline Regressio & & 63680085 & 21226695 & 169.91 & \\
$\mathrm{n}$ & 3 & .02 & .01 & 15 & 0.000113 \\
& & 499711.9 & 124927.9 & & \\
Residual & 4 & 057 & 764 & & \\
& & 64179796 & & & \\
Total & 7 & .93 & & & \\
\hline
\end{tabular}

\begin{tabular}{|c|c|c|c|c|c|c|c|c|c|}
\hline & & $\begin{array}{l}\text { Coefficien } \\
\text { ts }\end{array}$ & $\begin{array}{l}\text { Standard } \\
\text { Error }\end{array}$ & $\mathrm{t}$ Stat & $\begin{array}{l}\mathrm{P}- \\
\text { value }\end{array}$ & $\begin{array}{l}\text { Lower } \\
95 \%\end{array}$ & $\begin{array}{l}\text { Upper } \\
95 \%\end{array}$ & $\begin{array}{l}\text { Lower } \\
95.0 \%\end{array}$ & $\begin{array}{l}\text { Upper } \\
95.0 \%\end{array}$ \\
\hline & Intercept & $\begin{array}{l}2120.165 \\
227\end{array}$ & $\begin{array}{l}997.9543 \\
441\end{array}$ & $\begin{array}{l}2.124511 \\
246\end{array}$ & $\begin{array}{l}0.1008 \\
29\end{array}$ & -650.6 & $\begin{array}{l}4890.9 \\
31\end{array}$ & -650.6 & $\begin{array}{l}4890.9 \\
31\end{array}$ \\
\hline $\begin{array}{l}\text { Investm } \\
\text { ent }\end{array}$ & $\begin{array}{l}15444370 \\
800\end{array}$ & $\begin{array}{l}2.54021 \mathrm{E} \\
-08\end{array}$ & $\begin{array}{l}1.37109 \mathrm{E} \\
-08\end{array}$ & $\begin{array}{l}1.852686 \\
209\end{array}$ & $\begin{array}{l}0.1375 \\
61\end{array}$ & -1.3E-08 & $\begin{array}{l}6.35 \mathrm{E}- \\
08\end{array}$ & $\begin{array}{l}-1.3 \mathrm{E}- \\
08\end{array}$ & $\begin{array}{l}6.35 \mathrm{E}- \\
08\end{array}$ \\
\hline Exports & $\begin{array}{l}2.03415 \mathrm{E} \\
+11\end{array}$ & $\begin{array}{l}1.55563 E \\
-08\end{array}$ & $\begin{array}{l}3.21517 E \\
-09\end{array}$ & $\begin{array}{l}4.838394 \\
681\end{array}$ & $\begin{array}{l}0.0084 \\
1\end{array}$ & $6.63 \mathrm{E}-09$ & $\begin{array}{l}2.45 \mathrm{E}- \\
08\end{array}$ & $\begin{array}{l}6.63 \mathrm{E}- \\
09\end{array}$ & $\begin{array}{l}2.45 \mathrm{E}- \\
08\end{array}$ \\
\hline $\begin{array}{l}\text { Populati } \\
\text { on }\end{array}$ & $\begin{array}{l}- \\
0.5193304 \\
07\end{array}$ & $\begin{array}{l}2587.499 \\
119\end{array}$ & $\begin{array}{l}761.7578 \\
216\end{array}$ & $\begin{array}{l}3.396747 \\
687\end{array}$ & $\begin{array}{l}0.0273 \\
59\end{array}$ & 472.5203 & $\begin{array}{l}4702.4 \\
78\end{array}$ & $\begin{array}{l}472.52 \\
03\end{array}$ & $\begin{array}{l}4702.4 \\
78\end{array}$ \\
\hline
\end{tabular}

Source: (Calculation by Author)

Exhibit III: Regression output for India 


\begin{tabular}{ll} 
ISSN 2411-9563 (Print) \\
Regression Statistics \\
\hline Multiple R & \multicolumn{1}{l}{0.9909455} \\
& 92 \\
& 0.9819731 \\
R Square & 66 \\
Adjusted R & 0.9684530 \\
Square & 4 \\
Standard & 53.509184 \\
Error & 6 \\
Observatio & \\
ns & 8 \\
\hline
\end{tabular}

Regression Statistics

Multiple R $\quad 92$

R Square $\quad 66$

Adjusted R $\quad 0.9684530$

Square 4

Standard $\quad 53.509184$

Observatio

ANOVA

\begin{tabular}{|c|c|c|c|c|c|c|c|c|c|}
\hline & & $d f$ & SS & MS & $F$ & $\begin{array}{l}\text { Significan } \\
\text { ce F }\end{array}$ & & & \\
\hline & Regressio & & 623873.89 & & 72.630 & & & & \\
\hline & $\mathrm{n}$ & 3 & 23 & 207958 & 48 & 0.000606 & & & \\
\hline & & & 11452.931 & 2863.2 & & & & & \\
\hline & Residual & 4 & & 33 & & & & & \\
\hline & Total & 7 & $\begin{array}{l}635326.82 \\
36\end{array}$ & & & & & & \\
\hline & & $\begin{array}{l}\text { Coefficient } \\
s\end{array}$ & $\begin{array}{l}\text { Standard } \\
\text { Error }\end{array}$ & $\mathrm{t}$ Stat & $\begin{array}{l}\mathrm{P} \text { - } \\
\text { value }\end{array}$ & $\begin{array}{l}\text { Lower } \\
95 \%\end{array}$ & $\begin{array}{l}\text { Upper } \\
95 \%\end{array}$ & $\begin{array}{l}\text { Lower } \\
95.0 \%\end{array}$ & $\begin{array}{l}\text { Upper } \\
95.0 \%\end{array}$ \\
\hline & Intercept & $\begin{array}{l}2579.4223 \\
9\end{array}$ & $\begin{array}{l}1230.8387 \\
8\end{array}$ & $\begin{array}{l}2.0956 \\
62\end{array}$ & $\begin{array}{l}0.1041 \\
62\end{array}$ & -837.934 & $\begin{array}{l}5996.7 \\
79\end{array}$ & $\begin{array}{l}- \\
837.9 \\
34\end{array}$ & $\begin{array}{l}5996.7 \\
79\end{array}$ \\
\hline $\begin{array}{l}\text { Investm } \\
\text { ent }\end{array}$ & & $\begin{array}{l}5.39103 \mathrm{E}- \\
09\end{array}$ & $\begin{array}{l}3.83081 \mathrm{E}- \\
09\end{array}$ & $\begin{array}{l}1.4072 \\
84\end{array}$ & $\begin{array}{l}0.2320 \\
93\end{array}$ & $-5.2 \mathrm{E}-09$ & $\begin{array}{l}1.6 \mathrm{E}- \\
08\end{array}$ & $\begin{array}{l}-5.2 \mathrm{E}- \\
09\end{array}$ & $\begin{array}{l}1.6 \mathrm{E}- \\
08\end{array}$ \\
\hline Exports & $\begin{array}{l}1.26648 \mathrm{E} \\
+11\end{array}$ & $\begin{array}{l}2.05468 \mathrm{E}- \\
09\end{array}$ & $\begin{array}{l}4.8274 \mathrm{E}- \\
10\end{array}$ & $\begin{array}{l}4.2562 \\
8\end{array}$ & $\begin{array}{l}0.0130 \\
92\end{array}$ & 7.14E-10 & $\begin{array}{l}3.39 \mathrm{E}- \\
09\end{array}$ & $\begin{array}{l}7.14 \mathrm{E} \\
-10\end{array}$ & $\begin{array}{l}3.39 \mathrm{E}- \\
09\end{array}$ \\
\hline Populati & & - & & - & & & & - & \\
\hline on & $\begin{array}{l}1.5278148 \\
47\end{array}$ & $\begin{array}{l}1466.2667 \\
58\end{array}$ & $\begin{array}{l}798.37984 \\
22\end{array}$ & $\begin{array}{l}1.8365 \\
5\end{array}$ & $\begin{array}{l}0.1401 \\
58\end{array}$ & -3682.92 & $\begin{array}{l}750.39 \\
1\end{array}$ & $\begin{array}{l}3682 . \\
92\end{array}$ & $\begin{array}{l}750.39 \\
1\end{array}$ \\
\hline
\end{tabular}

Source: (Calculation by Author)

Exhibit IV: Regression output for China (PRC)

SUMMARY OUTPUT

Regression Statistics 


\begin{tabular}{|c|c|c|c|c|c|c|c|c|c|}
\hline & Multiple R & 0.974329 & & & & & & & \\
\hline & R Square & 0.949317 & & & & & & & \\
\hline & $\begin{array}{l}\text { Adjusted R } \\
\text { Square }\end{array}$ & 0.911305 & & & & & & & \\
\hline & $\begin{array}{l}\text { Standard } \\
\text { Error }\end{array}$ & 464.4933 & & & & & & & \\
\hline & $\begin{array}{l}\text { Observatio } \\
\text { ns }\end{array}$ & 8 & & & & & & & \\
\hline & ANOVA & & & & & & & & \\
\hline & & $d f$ & SS & MS & $F$ & $\begin{array}{l}\text { Significan } \\
\text { ce F }\end{array}$ & & & \\
\hline & Regression & 3 & $\begin{array}{l}161647 \\
54\end{array}$ & $\begin{array}{l}538825 \\
1\end{array}$ & $\begin{array}{l}24.974 \\
05\end{array}$ & 0.004734 & & & \\
\hline & Residual & 4 & 863016 & 215754 & & & & & \\
\hline & Total & 7 & $\begin{array}{l}170277 \\
70\end{array}$ & & & & & & \\
\hline & & $\begin{array}{l}\text { Coefficie } \\
\text { nts }\end{array}$ & $\begin{array}{l}\text { Standar } \\
\text { d Error }\end{array}$ & $\mathrm{t}$ Stat & P-value & $\begin{array}{l}\text { Lower } \\
95 \%\end{array}$ & $\begin{array}{l}\text { Upper } \\
95 \%\end{array}$ & $\begin{array}{l}\text { Lower } \\
95.0 \%\end{array}$ & $\begin{array}{l}\text { Upper } \\
95.0 \%\end{array}$ \\
\hline & Intercept & 2104.312 & $\begin{array}{l}5783.62 \\
4\end{array}$ & $\begin{array}{l}0.3638 \\
4\end{array}$ & $\begin{array}{l}0.7343 \\
93\end{array}$ & -13953.6 & $\begin{array}{l}18162 . \\
23\end{array}$ & $\begin{array}{l}- \\
13953 \\
.6\end{array}$ & $\begin{array}{l}18162 . \\
23\end{array}$ \\
\hline $\begin{array}{l}\text { Investme } \\
\mathrm{nt}\end{array}$ & $\begin{array}{l}621080430 \\
01\end{array}$ & $-2.1 \mathrm{E}-09$ & $\begin{array}{l}8.01 \mathrm{E}- \\
09\end{array}$ & $\begin{array}{l}- \\
0.2576 \\
2\end{array}$ & $\begin{array}{l}0.8094 \\
1\end{array}$ & $-2.4 \mathrm{E}-08$ & $\begin{array}{l}2.02 \mathrm{E}- \\
08\end{array}$ & $\begin{array}{l}-2.4 \mathrm{E}- \\
08\end{array}$ & $\begin{array}{l}2.02 \mathrm{E}- \\
08\end{array}$ \\
\hline Exports & $\begin{array}{l}6.55827 \mathrm{E}+ \\
11\end{array}$ & 3.03E-09 & 1.3E-09 & $\begin{array}{l}2.3278 \\
19\end{array}$ & $\begin{array}{l}0.0804 \\
44\end{array}$ & $-5.8 \mathrm{E}-10$ & $\begin{array}{l}6.64 \mathrm{E}- \\
09\end{array}$ & $\begin{array}{l}-5.8 \mathrm{E}- \\
10\end{array}$ & $\begin{array}{l}6.64 \mathrm{E}- \\
09\end{array}$ \\
\hline $\begin{array}{l}\text { Populati } \\
\text { on }\end{array}$ & $\begin{array}{l}0.5939328 \\
15\end{array}$ & -5154.42 & $\begin{array}{l}9221.40 \\
7\end{array}$ & $\begin{array}{l}- \\
0.5589 \\
6\end{array}$ & 0.606 & -30757.2 & $\begin{array}{l}20448 . \\
3\end{array}$ & $\begin{array}{l}- \\
30757 \\
.2\end{array}$ & $\begin{array}{l}20448 . \\
3\end{array}$ \\
\hline
\end{tabular}

Source: (Calculation by Author)

Exhibit V: Regression output for Brazil

SUMMARY OUTPUT

\begin{tabular}{ll}
\hline \multicolumn{3}{l}{ Regression Statistics } \\
\hline Multiple R & 0.980281 \\
R Square & 0.960952 \\
Adjusted R & \\
Square & 0.931665
\end{tabular}






Source: (Calculation by Author)

\section{Discussion}

As can be seen from the regression analysis in the case of South Africa, the R square was at 0.92 or $92 \%$ meaning investment, exports, and population growth explained GDP per capita. It means $8 \%$ of GDP per capita was explained by other factors not included in the analysis. The Anova $p$ value .013 shows that for South Africa INV, POP, and EXP are statistically significantly different from GDP per capita. However, from the regression output per se the same three variables do not show any predictive significance to GDP per capita. It means, therefore, they have not significantly contributed to GDP per capita growth.

In the case of Russia, the R square 0.99, it indicates that POP, INV, and EXP explain 99\% of the GDP per capita. Also Anova at significance level of .000113 indicates all the economic indicators selected have equal variance. Then, the $p$ values in the regression output illustrates that INV $(0.14)$ does not significantly predict GDP per capita. However, EXP $(0.008)$ and POP (0.03) have significantly predicted GDP per capita.

Moving on to India $R$ square at 0.98 shows $98 \%$ of the cases for EXP, POP, and INV explain the GDP. Then, the Anova at .0006 indicates a strong statistical significant difference among the same variables to GDP per capita. However, in the main regression it is only for $\operatorname{EXP}(.013)$ that there is indication that it significantly predicted GDP per capita. The same is not the case for INV (0.23) and POP (0.14). 
In addition, China has an R square of 0.95 meaning $95 \%$ of its INV, EXP, and POP is what explained the trend in GDP. With the Anova at .005 it shows a significant statistical difference between the GDP per capita and the rest three variables mentioned. However, none of the three independent economic variables significantly predicted the GDP.

Finally, for Brazil the R square was at 0.96 meaning $96 \%$ of the cases for EXP, POP, and INV did explain GDP per capita. The Anova with significance level at .003 shows a statistical significant difference between GDP and the three variables. However, in the main regression none of the three independent economic variables significantly predicted GDP.

It is worth stressing that for both China and Brazil EXP with 0.08 and 0.06 significance level were the closest in terms of significantly predicting the GDP compared to POP and INV.

\section{Conclusion}

In conclusion, it can now be confirmed that for BRICS POP, INV, and EXP explained over $90 \%$ of the GDP per capita. This is an important revelation from the regression analysis because it shows the three variables have been playing a role in economic development. But, when benchmarked with other independent economic variables it was seen that EXP was the highest variable in terms of significantly predicting GDP per capita. All, in all, in a future study it would be essential to assess data representing the BRICS region; it would be helpful to address the issue in this study using panel data analysis as a future improvement of the current outcomes of the study.

\section{References}

[1] Ekmekçioğlu, E. (2014). "Role of Financial Markets in International Trade and Brief Case Study of BRICS". International Journal of Economy, Management and Social Sciences, Vol 3 - № 6.

[2] Liu, X., Burridge, P. and Sinclairs, P.J.N. (2002). "Relationship between economic growth, foreign direct investment and trade: evidence from China". Applied Economics, Vol. 34 No. 11, pp. 1433-40.

[3] Makki, S. and Somwap, A. (2004). "Impact of foreign direct investment and trade on economic growth: evidence from developing countries". Journal of American Agricultural Economics, Vol. 8, pp. 795-801.

[4] Smith, J. (2011). BRIC becomes BRICS: Emerging Regional Powers? Changes on the Geopolitical Chessboard. Global Research Center on Globalisation. [ONLINE] Available at: http://www.globalresearch.ca/bric-becomesbrics-emerging-regional-powers-changes-on-the-geopolitical-chessboard/22812. [Accessed on 08.06.2014]

(APPENDIX) 
Table 1

\begin{tabular}{|c|c|c|c|c|c|c|c|c|c|}
\hline Indicator (South Africa) & Year 2004 & Year 2005 & Year 2006 & Year 2007 & Year 2008 & Year 2009 & Year 2010 & Year 2011 & Year 2012 \\
\hline Foreign direct investment, net inflows (BoP, current US\$) & 701422007,63 & 6522098178,18 & 623291744,34 & 6586792253,11 & 9885001293,44 & 7624489973,88 & 3693271715,48 & 4139289122,69 & 4643830666,02 \\
\hline Exports of goods and services (current US\$) & 57889752492,09 & 67643778942,06 & 78317824613,99 & 90077093237,89 & 98005339806,41 & 77556911881,05 & 99398844053,81 & 117659751183,48 & 108595055663,44 \\
\hline Population growth (annual \%) & 1,306274645 & 1,310203612 & 1,314170806 & 1,318181247 & 1,322245438 & 1,326361 & 1,330539186 & 1,334782477 & 1,339096709 \\
\hline GDP per capita (current USS) & 4659,623355 & 5185,849388 & 5407,258649 & 5850,958488 & 5511,195079 & 5654,492378 & 7136,963299 & 7789,944597 & 7351,756648 \\
\hline & & & & & & & & & \\
\hline Indicators (Russia) & Year 2004 & Year 2005 & Year 2006 & Year 2007 & Year 2008 & Year 2009 & Year 2010 & Year 2011 & Year 2012 \\
\hline Foreign direct investment, net inflows (BoP, current US\$) & 15444370800,00 & 15508100000,00 & 375947000000,00 & 55873700000,00 & 747829000000,00 & 36583100000,00 & 43167700000,00 & 55083600000,00 & 506610000000,00 \\
\hline Expotts of goods and services (current US\$) & 203415480735,86 & 268951741205,59 & 333908278474,50 & 392044033024,77 & 520003701781,28 & 341584673112,73 & 445512964893,39 & 576567394209,12 & 593112327003,54 \\
\hline GDP per capita (current USS) & 4108,56 & 5337,07 & 6946,88 & 9146,42 & 11700,22 & 8615,66 & 10709,51 & 13284,03 & 14037,02 \\
\hline Population growth (annual \%) & $-0,52$ & $-0,49$ & $-0,46$ & $-0,28$ & $-0,11$ & $-0,03$ & 0,34 & 0,40 & 0,40 \\
\hline & & & & & & & & & \\
\hline Indicators (India) & Year 2004 & Year 2005 & Year 2006 & Year 2007 & Year 2008 & Year 2009 & Year 2010 & Year 2011 & Year 2012 \\
\hline Foreign direct investment, net inflows (BoP, current US\$) & & $-4628652265,00$ & $-5992285935,00$ & $-8201628958,00$ & $-24149749830,00$ & $-19485789183,00$ & $-11428785746,90$ & $-23890659988,00$ & $-15442447343,00$ \\
\hline Exports of goods and services (current US\$) & 126647860366,00 & 160837896187,16 & 199973922363,78 & 253077528108,49 & 288901732847,28 & 273752028721,78 & 375351687886,03 & 448717644624,29 & 446027703580,27 \\
\hline GDP per capita (current USS) & 649,71 & 740,11 & 830,16 & 1068,68 & 1042,08 & 1147,24 & 1417,07 & 1539,60 & 1503,00 \\
\hline Population growth (annual \%) & 1,53 & 1,48 & 1,42 & 1,37 & 1,33 & 1,31 & 1,29 & 1,28 & 1,26 \\
\hline & & & & & & & & & \\
\hline Indicators (China) & Year 2004 & Year 2005 & Year 2006 & Year 2007 & Year 2008 & Year 2009 & Year 2010 & Year 2011 & Year 2012 \\
\hline Foreign direct investment, net inflows (BoP, current US\$) & 62108043001,00 & 104108693867,00 & 124082035619,00 & 156249335203,00 & 171534650312,00 & 131057052870,00 & 243703434558,00 & 280072219150,00 & 253474944300,00 \\
\hline Expots of goods and services (current US\$) & 655826577000,00 & 836887800000,00 & 1061681000000,00 & 13422060000000,00 & 1581713000000,00 & 13333000000000,00 & 1743366950035,36 & 2089357369251,35 & 2248376523481,44 \\
\hline GDP per capita (current USS) & 1490,38 & 1731,13 & 2069,34 & 2651,26 & 3413,59 & 3749,27 & 4433,36 & 5447,34 & 6091,01 \\
\hline Population growth (annual \%) & 0,59 & 0,59 & 0,56 & 0,52 & 0,51 & 0,50 & 0,48 & 0,48 & 0,49 \\
\hline & & & & & & & & & \\
\hline & & & & & & & & & \\
\hline Indicators (Brazil) & Year 2004 & Year 2005 & Year 2006 & Year 2007 & Year 2008 & Year 2009 & Year 2010 & Year 2011 & Year 2012 \\
\hline Foreign direct investment, net inflows (BoP, current US\$) & & $-12549590735,00$ & 9420275577,00 & $-27518241273,00$ & $-24601090274,00$ & $-36032806300,00$ & $-36918923577,00$ & $-67689141256,00$ & $-68093253945,00$ \\
\hline Expots of goods and services (current US\$) & 109023247863,25 & 133460147904,68 & 156460477941,18 & 182671465929,37 & 225926235617,04 & 177877857668,03 & 232981910479,97 & 294453331491,45 & 282874928219,53 \\
\hline GDP per capita (current USS) & 3607,19 & 4739,31 & 5787,98 & 7194,08 & 8622,71 & 8373,34 & 10978,09 & 12575,98 & 11339,52 \\
\hline Population growth (annual \%) & 1,23 & 1,15 & 1,06 & 0,99 & 0,93 & 0,90 & 0,88 & 0,88 & 0,87 \\
\hline
\end{tabular}

